\title{
GLOBULAR CLUSTER AGES FROM HIPPARCOS SUBDWARFS
}

\author{
F. PONT ${ }^{1}$, D.A. VANDENBERG ${ }^{2}$, M. MAYOR ${ }^{1}$ AND C. TURON $^{3}$ \\ 1 Observatoire de Genève, 1290 Sauverny, Switzerland \\ ${ }^{2}$ Dpt. of Physics and Astronomy, University of Victoria, Canada \\ 3 DASGAL, Observatoire de Paris-Meudon, France
}
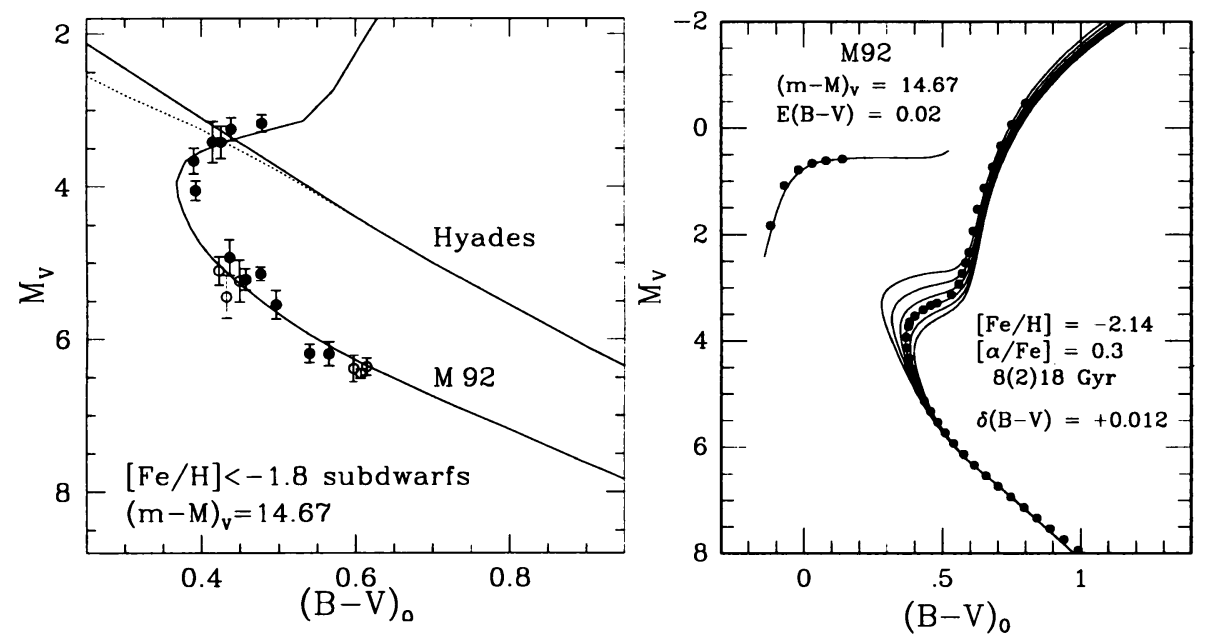

The new Hipparcos parallax data for local subdwarfs allow a much more reliable determination of the distance to globular clusters (by direct sequence fitting) than was previously possible. Earlier studies (Reid 1997, Gratton et al. 1997) have reported larger distances than expected, especially for the most metal-poor clusters, implying much younger ages. In our study of Hipparcos subdwarfs applied to M92 - representative of the oldest, most metal-poor clusters - we find however a distance only slightly in excess of previous expectations. We show, using Monte Carlo simulations, that most of the difference may be explained by our more detailed treatment of the Lutz-Kelker and selection biases. With up-to-date stellar evolution models, we derive a minimum age of $13 \mathrm{Gyr}$ for the Universe. This value, although lower than previous estimates, still imposes a rather strict upper limit to $H_{o}$ in the context of standard cosmological models.

(Results published in Pont et al. 1997, A\&A; or http://obswww.unige.ch/ $\sim$ pont)

K. Sato (ed.), Cosmological Parameters and the Evolution of the Universe, 69.

(C) 1999 IAU. Printed in the Netherlands. 